\title{
Decoy-state quantum key distribution with more than three types of photon intensity pulses
}

\author{
H. F. Chau* \\ Department of Physics and Center of Theoretical and Computational Phyics, University of Hong Kong, Pokfulam Road, Hong Kong
}

(Received 10 December 2017; published 2 April 2018)

\begin{abstract}
The decoy-state method closes source security loopholes in quantum key distribution (QKD) using a laser source. In this method, accurate estimates of the detection rates of vacuum and single-photon events plus the error rate of single-photon events are needed to give a good enough lower bound of the secret key rate. Nonetheless, the current estimation method for these detection and error rates, which uses three types of photon intensities, is accurate up to about $1 \%$ relative error. Here I report an experimentally feasible way that greatly improves these estimates and hence increases the one-way key rate of the BB84 QKD protocol with unbiased bases selection by at least $20 \%$ on average in realistic settings. The major tricks are the use of more than three types of photon intensities plus the fact that estimating bounds of the above detection and error rates is numerically stable, although these bounds are related to the inversion of a high condition number matrix.
\end{abstract}

DOI: 10.1103/PhysRevA.97.040301

In quantum key distribution (QKD), two trusted parties, Alice and Bob, share a secret key via preparation and measurement of photons transmitted through a noisy channel controlled by an eavesdropper, Eve. Most QKD experiments to date employ phase-randomized Poissonian distributed photon sources to generate photons at a reasonably high rate and use the decoy-state method to tackle Eve's photon-number-splitting attack on multiple photon events emitted from the Poissonian sources. (See, for example, Ref. [1] for an overview.) The key idea of the decoy-state method is that although Eve knows the photon number in each pulse, she does not know the probability distribution of photon number from which the pulse is drawn. So, by preparing each photon pulse independently from a collection of Poissonian sources with different intensity parameters (in other words, different average photon number per pulse), Alice and Bob may obtain a lower bound of the key rate of the final secret key they share $[2,3]$. The decoy-state technique can handle a variety of QKD protocols including those with two-way classical postprocessing [4], those involving the transmission of qudits [5], and those with finite raw key length [6].

However, the state-of-the-art method to date, which employs a Poissonian source with three different types of intensities - one high, one weak, and one equal or close to zero intensity—is inefficient for two reasons. First, using weak and zero intensity photon pulses lowers the average photon transmission rate. Second, the provably secure decoy-state key rate depends on good enough lower bounds on parameters $Y_{\mathrm{X}, 0}, Y_{\mathrm{X}, 1}, Y_{\mathrm{Z}, 1}$ together with a good upper bound on $e_{\mathrm{Z}, 1}$ to be defined later. Nonetheless, I am going to report later in the Rapid Communication that the bounds of these four parameters obtained through the state-of-the-art method used in Refs. [2,3,6,7] deviate from their actual values with an average relative erros of $\approx 1 \%$ over a set of randomly chosen quantum channels.

\footnotetext{
*hfchau@hku.hk
}

Here I show how to perform decoy-state QKD using higher average photon intensity pulses plus refined upper and lower bounds on the above four parameters to give a much improved provably secure key rate in realistic settings used in typical QKD experiments with finite raw key length. The main ingredients used here are the use of more than three different types of photon intensities plus the observation that the lower and upper bounds obtained are numerically stable, although one has to effectively invert a large condition number matrix. I illustrate the key idea using a specific BB84 QKD protocol [8] which uses the $\mathrm{X}$ measurement results to generate the raw key and the $\mathrm{Z}$ measurement results to check the channel phase error here. It is straightforward to extend the analysis to other QKD schemes such as the six-state scheme and some qudit-based schemes $[9,10]$.

Suppose Alice and Bob use $k \geqslant 2$ different photon intensities labeled $\mu_{1}>\mu_{2}>\cdots>\mu_{k} \geqslant 0$ with probabilities $p_{\mu_{1}}, \ldots, p_{\mu_{k}}$. The observed average yield per photon pulse prepared in the $\mathrm{B}=\mathrm{X}$ and $\mathrm{Z}$ bases using intensity $\mu_{n}$ is given by $[2,3,7]$

$$
Q_{\mathrm{B}, \mu_{n}}=\sum_{m=0}^{+\infty} \frac{\mu_{n}^{m} Y_{\mathrm{B}, m} \exp \left(-\mu_{n}\right)}{m !},
$$

where $Y_{\mathrm{B}, m}$ is the probability of photon detection by Bob given that the photon pulse sent by Alice contains $m$ photons. (From now on, B denotes either $\mathrm{X}$ or Z.) Similarly, the observed average bit error rate $E_{\mathrm{B}, \mu_{n}}$ is given by

$$
Q_{\mathrm{B}, \mu_{n}} E_{\mathrm{B}, \mu_{n}}=\sum_{m=0}^{+\infty} \frac{\mu_{n}^{m} Y_{\mathrm{B}, m} e_{\mathrm{B}, m} \exp \left(-\mu_{n}\right)}{m !},
$$

where $e_{\mathrm{B}, m}$ is the bit error rate for $m$ photon emission events prepared in the $\mathrm{B}$ basis.

As $Y_{\mathrm{B}, m}$ and $e_{\mathrm{B}, m}$ are independent of the intensity parameter $\mu_{n}$ used, they can be in estimated or bounded by solving Eqs. (1) and (2) from a collection of intensity parameters $\mu_{n}$. Numerical stability issue aside, infinitely many intensities $\mu_{n}$ are needed to determine all the $Y_{\mathrm{B}, m}$ 's and $e_{\mathrm{B}, m}$ 's. Fortunately, 
they only need to know lower bounds of $Y_{\mathrm{X}, 0}, Y_{\mathrm{X}, 1}$ and an upper bound of $H_{2}\left(e_{\mathrm{Z}, 1}\right)$ to give a lower bound of the one-way secret key rate $R$ of the BB84 QKD protocol with the raw key all coming from $\mathrm{X}$ measurements. This is because the secret key rate $R$, which is defined as the number of final secret bits divided by the expected number of photon pulses sent through the channel, is given by [6]

$$
\begin{aligned}
R= & p_{\mathrm{X}}^{2}\left\{\langle\exp (-\mu)\rangle Y_{\mathrm{X}, 0}+\langle\mu \exp (-\mu)\rangle Y_{\mathrm{X}, 1}\left[1-H_{2}\left(e_{p}\right)\right]\right. \\
& \left.-\left\langle Q_{\mathrm{X}, \mu} H_{2}\left(E_{\mathrm{X}, \mu}\right)\right\rangle-\frac{\left\langle Q_{\mathrm{X}, \mu}\right\rangle}{\ell_{\mathrm{raw}}}\left[6 \log _{2} \frac{\chi(k)}{\epsilon_{\mathrm{sec}}}+\log _{2} \frac{2}{\epsilon_{\mathrm{cor}}}\right]\right\} .
\end{aligned}
$$

Here $p_{\mathrm{X}}$ is the chance that Alice (Bob) uses $\mathrm{X}$ as the preparation (measurement) basis, the symbol $\langle f(\mu)\rangle$ denotes $\sum_{n=1}^{k} p_{\mu_{n}} f\left(\mu_{n}\right), H_{2}(x) \equiv-x \log _{2} x-(1-x) \log _{2}(1-x)$ is the binary entropy function, $e_{p}$ is the phase error rate of the single-photon events in the raw key, and $\ell_{\text {raw }}$ is the length of the raw sifted key bits. For BB84, $e_{p} \rightarrow e_{\mathrm{Z}, 1}$ as $\ell_{\text {raw }} \rightarrow+\infty$. Also, the probability that the final secret keys shared between Alice and Bob are different is at most $\epsilon_{\mathrm{cor}}$, Eve's information on the final key is at most $\epsilon_{\sec }$ [11-13], and $\chi(k)$ is a QKD scheme specific factor depending on the number of photon intensities $k$ as well as the detailed security analysis used. For the case studied by Lim et al. in Ref. [6], $\chi(3)=21$.

We shall see from Eq. (4) below, the current method of getting an upper bound for $e_{\mathrm{Z}, 1}$ requires knowledge of the lower bounds of $Y_{\mathrm{z}, 0}$ and $Y_{\mathrm{z}, 1}$ plus an upper bound of $Y_{\mathrm{z}, 1} e_{\mathrm{z}, 1}$ [6,7]. The goal, therefore, is to determine the bounds for the five parameters $-Y_{\mathrm{B}, m}(\mathrm{~B}=\mathrm{X}$ and $\mathrm{Z}, m=0,1)$ and $e_{\mathrm{Z}, 1}$-as close to their actual values as possible using finite types of photon intensities $k$ (and hence a finite number of $Q_{\mathrm{B}, \mu_{n}}$ 's and $Q_{\mathrm{B}, \mu_{n}} E_{\mathrm{B}, \mu_{n}}$ 's). The current method uses three different intensities $\mu_{1}>\mu_{2}>\mu_{3} \geqslant 0$ and the corresponding bounds are given by

$$
\begin{aligned}
& Y_{\mathrm{B}, 0} \geqslant \frac{\mu_{2} Q_{\mathrm{B}, \mu_{3}}^{\langle\langle 1\rangle\rangle} \exp \left(\mu_{3}\right)-\mu_{3} Q_{\mathrm{B}, \mu_{2}}^{\langle\langle 0\rangle\rangle} \exp \left(\mu_{2}\right)}{\mu_{2}-\mu_{3}}, \\
& Y_{\mathrm{B}, 1} e_{\mathrm{B}, 1} \\
& \leqslant \frac{\left(Q_{\mathrm{B}, \mu_{2}} E_{\mathrm{B}, \mu_{2}}\right)^{\langle\langle 0\rangle\rangle} \exp \left(\mu_{2}\right)-\left(Q_{\mathrm{B}, \mu_{3}} E_{\mathrm{B}, \mu_{3}}\right)^{\langle\langle 1\rangle\rangle} \exp \left(\mu_{3}\right)}{\mu_{2}-\mu_{3}},
\end{aligned}
$$

and

$$
\begin{aligned}
Y_{\mathrm{B}, 1} \geqslant & \frac{\mu_{1}}{\mu_{1}\left(\mu_{2}-\mu_{3}\right)-\mu_{2}^{2}+\mu_{3}^{2}}\left\{Q_{\mathrm{B}, \mu_{2}}^{\langle\langle 1\rangle\rangle} \exp \left(\mu_{2}\right)-Q_{\mathrm{B}, \mu_{3}}^{\langle\langle 0\rangle\rangle}\right. \\
& \left.\times \exp \left(\mu_{3}\right)+\frac{\left(\mu_{2}^{2}-\mu_{3}^{2}\right)\left[Y_{\mathrm{B}, 0}-Q_{\mathrm{B}, \mu_{1}}^{\langle\langle 0\rangle\rangle} \exp \left(\mu_{1}\right)\right]}{\mu_{1}^{2}}\right\}
\end{aligned}
$$

provided that $\mu_{1}>\mu_{2}+\mu_{3}$ [6,7]. In the above equations, $Q_{\mathrm{B}, \mu_{n}}^{\langle\langle i\rangle\rangle}=Q_{\mathrm{B}, \mu_{n}}+(-1)^{i} \Delta Q_{\mathrm{B}, \mu_{n}}$ and $\left(Q_{\mathrm{B}, \mu_{n}} E_{\mathrm{B}, \mu_{n}}\right)^{\langle\langle i\rangle\rangle}=$ $Q_{\mathrm{B}, \mu_{n}} E_{\mathrm{B}, \mu_{n}}+(-1)^{i} \Delta\left(Q_{\mathrm{B}, \mu_{n}} E_{\mathrm{B}, \mu_{n}}\right)$, where $\Delta$-ed quantities are the upper bounds on the statistical fluctuation due to finite- size sampling. From the Hoeffding inequality [14], these fluctuations can be taken to be at most

$$
\Delta Q_{\mathrm{B}, \mu_{n}}=\frac{\left\langle Q_{\mathrm{B}, \mu}\right\rangle}{p_{\mu_{n}}}\left\{\frac{\ln \left[\frac{\chi(k)}{\epsilon_{\mathrm{sec}}}\right]}{2 s_{\mathrm{B}}}\right\}^{1 / 2}
$$

and

$$
\Delta\left(Q_{\mathrm{Z}, \mu_{n}} E_{\mathrm{Z}, \mu_{n}}\right)=\frac{1}{p_{\mu_{n}}}\left\{\frac{\left\langle Q_{\mathrm{Z}, \mu}\right\rangle\left\langle Q_{\mathrm{Z}, \mu} E_{\mathrm{Z}, \mu}\right\rangle \ln \left[\frac{\chi(k)}{\epsilon_{\mathrm{sec}}}\right]}{2 s_{\mathrm{Z}}}\right\}^{1 / 2},
$$

each with probability at least $1-\epsilon_{\mathrm{sec}} / \chi(k)$, where $s_{\mathrm{B}}$ is the number of bits that Alice prepares and Bob successfully measures in the B basis. Obviously, $s_{\mathrm{X}}=\ell_{\text {raw }}$ and $s_{\mathrm{Z}} \approx(1-$ $\left.p_{\mathrm{X}}\right)^{2} s_{\mathrm{X}} / p_{\mathrm{X}}^{2}$. Due to finite key length, the phase error rate of the single-photon events in the raw key is upper bounded by [15]

$$
\begin{gathered}
e_{p} \leqslant e_{\mathrm{Z}, 1}+\bar{\gamma}\left(\epsilon_{\mathrm{sec}} / \chi(k), e_{\mathrm{Z}, 1}, s_{\mathrm{Z}} Y_{\mathrm{Z}, 1}\langle\mu \exp (-\mu)\rangle /\left\langle Q_{\mathrm{Z}, \mu}\right\rangle\right. \\
\left.s_{\mathrm{X}} Y_{\mathrm{X}, 1}\langle\mu \exp (-\mu)\rangle /\left\langle Q_{\mathrm{X}, \mu}\right\rangle\right)
\end{gathered}
$$

with probability at least $1-\epsilon_{\mathrm{sec}} / \chi(k)$, where

$$
\bar{\gamma}(a, b, c, d) \equiv \sqrt{\frac{(c+d)(1-b) b}{c d} \ln \left[\frac{c+d}{2 \pi c d(1-b) b a^{2}}\right]} .
$$

This equation is based on the estimate in Ref. [6], which in turn is deduced from Eqs. (18) and (22) in Ref. [15]. However, the factor $1 / \sqrt{2 \pi}$ is omitted in Eq. (18); and the $\ln 2$ factor in Eq. (22) should be in the denominator. The $\bar{\gamma}$ above is deducted using the same way as in Ref. [15] with the corrected equations. Note further that $\bar{\gamma}$ is ill-defined if $a, c, d$ are too large. This is because in such case no $e_{p} \geqslant e_{\mathrm{Z}, 1}$ exists with failure probability $a$. All parameters used in this Rapid Communication are carefully picked so that $\bar{\gamma}$ is well defined.

Interestingly, the $Y_{\mathrm{B}, 0}$ bound is tight when $\mu_{3} \rightarrow 0[6,7]$. (Similar bounds have been reported in Refs. [2,16].) As for $\mu_{1}$ and $\mu_{2}$, they cannot be too close to each other in practice. Otherwise, the $Y_{\mathrm{B}, 1}$ bound may not be reliable in the case of finite key length [2]. In most experiments to date, the key rate $R$ is optimized by choosing $\mu_{1} \gtrsim 0.5,0.01 \lesssim \mu_{2} \lesssim 0.1$, and $\mu_{3} \approx 0$ [17-21].

While this choice of photon intensities can accurately determine the value of $Y_{\mathrm{B}, 0}$, it is not very good at estimating $Y_{\mathrm{B}, 1}$ and $e_{\mathrm{Z}, 1}$. Using the above photon intensities, ignoring finite key length effect, and by randomly picking $Y_{\mathrm{B}, m}$ 's in $[0,1]$ and $e_{\mathrm{Z}, m}$ 's in $[0,0.5]$, I numerically find from Eq. (4) that the average (maximum) relative errors of the estimated $Y_{\mathrm{B}, 1}$ and $Y_{\mathrm{X}, 1} H_{2}\left(e_{p}\right)$ from their true values can be as high as $\approx 1 \%$ $(\approx 5 \%)$. The deviation of the estimated $Y_{\mathrm{B}, 1}$ from its actual value increases as $\mu_{1}$ or $\mu_{2}$ increase; and the deviation of the estimated $Y_{\mathrm{X}, 1} H_{2}\left(e_{p}\right)$ from its actual value increases as $\mu_{1}$ or $e_{\mathrm{Z}, 1}$ increase. Consequently, Alice and Bob face a dilemma. Using small $\mu_{1}$ and/or $\mu_{2}$ give much better estimates of $Y_{\mathrm{B}, 1}$ and $Y_{\mathrm{X}, 1} H_{2}\left(e_{p}\right)$ at the expense of a lower raw key generation rate and hence a lower $R$. This is particularly true in the practical situation of a finite raw key length of $\lesssim 10^{9}$ to $10^{10}$ bits as using biased choice of photon intensities $p_{\mu_{i}}$ 's cannot increase the key rate too much. 
Now, I show how to obtain a higher key rate by using a few larger $\mu_{n}$ 's and better estimates for $Y_{\mathrm{B}, 1}$ and $Y_{\mathrm{X}, 1} H_{2}\left(e_{p}\right)$. The trick is to directly solve Eqs. (1) and (2) for $k \geqslant 2$ different photon intensities. I rewrite Eq. (2) as

$$
\begin{aligned}
Y_{\mathrm{B}, m} & =\sum_{i=1}^{k}\left(M^{-1}\right)_{m+1, i}\left[Q_{\mathrm{B}, \mu_{i}} \exp \left(\mu_{i}\right)-\sum_{j=k}^{+\infty} \frac{\mu_{i}^{j} Y_{\mathrm{B}, j}}{j !}\right] \\
& \equiv \sum_{i=1}^{k}\left(M^{-1}\right)_{m+1, i} Q_{\mathrm{B}, \mu_{i}} \exp \left(\mu_{i}\right)+\sum_{j=k}^{+\infty} C_{m+1, j} Y_{\mathrm{B}, j}
\end{aligned}
$$

for $m=0,1, \ldots, k-1$, where $M_{i j}=\mu_{i}^{j-1} /(j-1)$ ! for $1 \leqslant$ $i, j \leqslant k$. In this way, I may express $Y_{\mathrm{B}, 0}, Y_{\mathrm{B}, 1}, \ldots, Y_{\mathrm{B}, k-1}$ in terms of $Q_{\mathrm{B}, \mu_{i}}$ 's for $i=1,2, \ldots, k, Y_{\mathrm{B}, j}$ 's for $j \geqslant k$, and the $k \times k$ matrix $M^{-1}$. Similarly, I use

$$
\begin{aligned}
Y_{\mathrm{Z}, m} e_{\mathrm{Z}, m}= & \sum_{i=1}^{k}\left(M^{-1}\right)_{m+1, i} Q_{\mathrm{z}, \mu_{i}} E_{\mathrm{Z}, \mu_{i}} \exp \left(\mu_{i}\right) \\
& +\sum_{j=k}^{+\infty} C_{m+1, j} Y_{\mathrm{Z}, j} e_{\mathrm{Z}, j}
\end{aligned}
$$

to solve $Y_{\mathrm{Z}, m} e_{\mathrm{Z}, m}$ for $m=0,1, \ldots, k-1$.

At first glance, this straightforward approach appears to be hopeless because accurate bounds of $Y_{\mathrm{B}, m}$ 's and $Y_{\mathrm{Z}, m} e_{\mathrm{Z}, m}$ 's $(m<k)$ require the number of photon intensities $k$ to be large. Nonetheless, large $k$ means that one has to invert the large condition number matrix $M$ so that the solutions of Eqs. (8) and (9) are sensitive to perturbation on $Y_{\mathrm{B}, j}$ 's and $Y_{\mathrm{Z}, j} e_{\mathrm{Z}, j}$ 's $(j \geqslant k)$ as well as uncertainty due to finite sample size for $Q_{\mathrm{B}, \mu_{i}}$ 's and $Q_{\mathrm{Z}, \mu_{i}} E_{\mathrm{Z}, \mu_{i}}$ 's. Numerical stability is an issue. Also, the bound on, say, $Y_{\mathrm{B}, 1}$ requires extremization over all $Y_{\mathrm{B}, j} \in[0,1]$ for $j \geqslant k$, which further complicates matters.

On second thought, sensitivity to perturbation and numerical stability are not relevant in computing the lower bound on $R$ provided that the four variables $Y_{\mathrm{X}, 0}, Y_{\mathrm{X}, 1}, Y_{\mathrm{z}, 1}$, and $Y_{\mathrm{Z}, 1} e_{\mathrm{Z}, 1}$ are insensitive to perturbation and numerically stable. I now report explicit expressions for the bounds on these four variables and then show that these expressions are indeed numerically stable.

Multiplying column $i$ of matrix $M$ by $\mu_{i}$ and row $j$ by $1 /(j-1)$ ! gives the corresponding Vandermonde's matrix. So I could use the explicit expression of the inverse of this Vandermonde's matrix in Ref. [22] to obtain

$$
\left(M^{-1}\right)_{m+1, i}=\frac{(-1)^{k-m-1} S_{i m} m !}{\prod_{t \neq i}\left(\mu_{i}-\mu_{t}\right)}
$$

for all $0 \leqslant m \leqslant k-1$. Here

$$
S_{i m}=\sum^{\prime} \mu_{t_{1}} \mu_{t_{2}} \cdots \mu_{t_{k-m-1}},
$$

where the primed sum is over all $1 \leqslant t_{1}<t_{2}<\cdots<$ $t_{k-m-1} \leqslant k$ with $t_{1}, t_{2}, \ldots, t_{k-m-1} \neq i$. Since $\left\{\mu_{i}\right\}_{i=1}^{k}$ is a strictly decreasing non-negative sequence, the sign of $\left(M^{-1}\right)_{m+1, i}$ equals $(-1)^{k-m-i}$. Thus, a lower bound of $Y_{\mathrm{B}, m}$ is obtained by replacing $Q_{\mathrm{B}, \mu_{i}}$ in the right-hand side of Eq. (8) by $Q_{\mathrm{B}, \mu_{i}}^{\langle\langle k-m-i-1\rangle)}$; and an upper bound of $Y_{\mathrm{Z}, 1} e_{\mathrm{Z}, 1}$ is obtained by replacing $Q_{\mathrm{Z}, \mu_{i}} E_{\mathrm{Z}, \mu_{i}}$ in the right-hand side of Eq. (9) by $\left(Q_{\mathrm{Z}, \mu_{i}} E_{\mathrm{Z}, \mu_{i}}\right)^{\langle\langle k-m-i\rangle\rangle}$. These bounds take care of the worst case deviations of measured $Q_{\mathrm{B}, \mu_{i}}$ 's and $Q_{\mathrm{Z}, \mu_{i}} E_{\mathrm{Z}, \mu_{i}}$ 's from their actual values due to finite sample size through the Hoeffding inequality.

As for the extremization of $Y_{\mathrm{B}, 0}$ and $Y_{\mathrm{B}, 1}$ over $Y_{\mathrm{B}, j}$ 's for $j \leqslant k$, I need to know the signs of $C_{m+1, j}$ for all $j \geqslant k$ defined in Eq. (8). In the Appendix, I show that $C_{1 j} \geqslant 0$ and $C_{2 j}<0$ $\left(C_{1 j} \leqslant 0\right.$ and $\left.C_{2 j}>0\right)$ if $k$ is even (odd). In both cases, extrema occur when $Y_{\mathrm{B}, j}=0$ or 1 for all $j \geqslant k$. For better estimation of the four variables $Y_{\mathrm{x}, 0}, Y_{\mathrm{x}, 1}, Y_{\mathrm{z}, 1}$, and $Y_{\mathrm{z}, 1} e_{\mathrm{Z}, 1}$, whose values are less than $1 / 2$ in essentially all practical situations, it makes sense to bound them via Eqs. (8) and (9) when the extrema occur when $Y_{\mathrm{B}, j}=Y_{\mathrm{Z}, j} e_{\mathrm{Z}, j}=0$ for all $j \geqslant k$. Hence, the lower bounds of $Y_{\mathrm{B}, 0}\left(Y_{\mathrm{B}, 1}\right)$ should be found from Eq. (8) by putting $Y_{\mathrm{B}, j}=0$ for all $j \geqslant k$ using $Q_{\mathrm{B}, \mu_{i}}$ 's taken from an even (odd) number of photon intensities; and the upper bound of $Y_{\mathrm{B}, 1} e_{\mathrm{Z}, 1}$ should be found from Eq. (9) by putting $Y_{\mathrm{z}, j} e_{\mathrm{z}, j}=0$ for all $j \geqslant k$ using $Q_{\mathrm{Z}, \mu_{i}} E_{\mathrm{Z}, \mu_{i}}$ 's taken from even number of photon intensities.

To obtain a better bound, more photon intensities can be used. To obtain a higher key rate $R$, some of the photon intensities should be as high as 1 . (If $\mu>1$, the chance of having a multiple photon event is too high that $R$ is compromised.) Nevertheless, deviations between the actual and measured values of $Q_{\mathrm{B}, \mu_{i}}$ 's increase as more photon intensities $k$ are used to obtain a raw key of a given length $\ell_{\text {raw }}$. These deviations may further amplify by $M^{-1}$ in Eqs. (8) and (9). My numerical finding suggests that four to five photon intensities seem to give optimal key rates using realistic parameters. Out of the $k$ photon intensities, I use data from the least $2\lfloor k / 2\rfloor$ of them to obtain the lower bound of $Y_{\mathrm{X}, 0}$ and the upper bound of $Y_{\mathrm{Z}, 1} e_{\mathrm{Z}, 1}$. And I use data from the least $2\lfloor(k-1) / 2\rfloor+1$ photon intensities to obtain the lower bound of $Y_{\mathrm{B}, 1}$. To sum up, the bounds I use are

$$
\begin{aligned}
& Y_{\mathrm{B}, 0} \geqslant \max \left(0, \sum_{i=k_{0}}^{k} \frac{-Q_{\mathrm{B}, \mu_{i}}^{\left\langle\left\langle k_{0}-i\right\rangle\right)} \exp \left[\mu_{i}\right] \hat{\prod}_{j \neq i} \mu_{j}}{\prod_{t \neq i}\left[\mu_{i}-\mu_{t}\right]}\right), \\
& Y_{\mathrm{B}, 1} \geqslant \max \left(0, \sum_{i=3-k_{0}}^{k} \frac{-Q_{\mathrm{B}, \mu_{i}}^{\left\langle\left\langle k_{0}-i\right\rangle\right\rangle} \exp \left[\mu_{i}\right] \hat{S}_{i}}{\hat{\prod}_{t \neq i}\left[\mu_{i}-\mu_{t}\right]}\right),
\end{aligned}
$$

and

$$
Y_{\mathrm{Z}, 1} e_{\mathrm{z}, 1} \leqslant \min \left(\frac{1}{2}, \sum_{i=k_{0}}^{k} \frac{\left[Q_{\mathrm{z}, \mu_{i}} E_{\mathrm{z}, \mu_{i}}\right]^{\left\langle\left\langle k_{0}-i\right\rangle\right\rangle} \exp \left[\mu_{i}\right] \hat{S}_{i}}{\hat{\prod}_{t \neq i}\left[\mu_{i}-\mu_{t}\right]}\right),
$$

where $k_{0}=1(2)$ if $k$ is even (odd), and $\hat{\prod}_{t \neq i}$ is over the dummy variable $t$ from $k_{0}$ to $k$ but skipping $i$. In addition, $\hat{S}_{i}=$ $\sum^{\prime \prime} \mu_{t_{1}} \mu_{t_{2}} \cdots \mu_{t_{k-k_{0}-1}}$ where the double primed sum is over $k_{0} \leqslant t_{1}<t_{2}<\cdots<t_{k-k_{0}-1} \leqslant k$ with $t_{1}, t_{2}, \ldots, t_{k-k_{0}-1} \neq i$. [I have added the trivial conditions in Eq. (12) to prevent the variables used in Eq. (3) from taking on absurd values.]

Interestingly, this method reduces to the bounds in Eq. (4) in the case of $k=3$ and $\mu_{3}=0$. More importantly, it is obvious from Eq. (12) that these bounds are numerically stable provided that the photon intensities $\mu_{i}$ are not close, say, with differences of at least $1 / 10$ so that the loss of precision in $1 /\left(\mu_{i}-\mu_{t}\right)$ is not serious even taking the intensity fluctuation in realistic source into consideration. (Intensity fluctuation of the order of $10^{-2}$ is 
easily attained in real experiments using a strong intensity and power stable laser plus suitable attenuators.) In contrast, it is clear that the $m$ ! factor in Eq. (10) is the origin of the numerical instability of finding $Y_{\mathrm{B}, m}$ 's for large $m$ 's.

I follow Ref. [6] by using the following security parameters: $\epsilon_{\text {cor }}=10^{-15}$ and $\epsilon_{\text {sec }}=\kappa \ell_{\text {final }}$, where $\ell_{\text {final }} \approx R s_{\mathrm{X}} /\left(p_{\mathrm{X}}^{2}\left\langle Q_{\mathrm{X}, \mu}\right\rangle\right)$ is the length of the final key and $\kappa=10^{-15}$ can be interpreted as the secrecy leakage per final secret bit. Following the derivation in Ref. [6], $\chi(k)=9+(4 k-2)$. [The term $4 k-2$ comes from $2 \times 2\lfloor k / 2\rfloor+2 \times[2\lfloor(k-1) / 2\rfloor+1]$, and the term 9 is independent of the number of photon intensities used. Note that using this method, $\chi(3)=19$, which is less than the $\chi$ used by the current method [6] by 2 . It gives a slightly higher $R$.]

I first study the key rate on a dedicated $100-\mathrm{km}$-long optical fiber system using the channel model in Ref. [6], whose channel parameters are deduced from the experiment in Ref. [23]. In this system, $Q_{\mathrm{B}, \mu} \approx\left(1+p_{\mathrm{ap}}\right)\left(2 p_{\mathrm{dc}}+\eta_{\mathrm{sys}} \mu\right)$ and $Q_{\mathrm{B}, \mu} E_{\mathrm{B}, \mu} \approx$ $\left(1+p_{\text {ap }}\right) p_{\mathrm{dc}}+\left(e_{\text {mis }} \eta_{\mathrm{ch}}+p_{\text {ap }} \eta_{\text {sys }} / 2\right) \mu$ for $0 \leqslant \mu \leqslant 1$, with after pulse probability $p_{\text {ap }}=4 \times 10^{-2}$, dark count probability $p_{\mathrm{dc}}=6 \times 10^{-7}$, error rate of the optical system $e_{\mathrm{mis}}=5 \times$ $10^{-3}$, and transmittances of the fiber and the system $\eta_{\mathrm{ch}}=$ $1 \times 10^{-2}$ and $\eta_{\text {sys }}=1 \times 10^{-3}$ [6]. Fixing $s_{\mathrm{X}}=10^{9}$ and the minimum photon intensity to $1 \times 10^{-6}$, while optimizing over $p_{\mathrm{X}}$ as well as all other photon intensities $\mu$ and all the $p_{\mu}$ 's, I find that the optimized one-way key rates for using $k=3,4,5$ equal $1.51 \times 10^{-5}, 1.57 \times 10^{-5}$, and $1.46 \times 10^{-5}$, respectively. That is to say, using $k=4$ in this case increases the key rate by more than about $4 \%$ over the standard $k=3$ method.

To further study the general performance on different channels, I compute the average key rate $\langle R\rangle$ over a random sample of uniformly and independently distributed $Y_{\mathrm{B}, m} \in\left[0, Y_{\max }\right]$ and $e_{\mathrm{B}, m} \in\left[0, e_{\max }\right]$ for all $m \geqslant 0$. [But I set $e_{\mathrm{B}, 0}=1 / 2$ as this is the basic assumption on the detector used. I also set $R=0$ for those sample channels whose key rates from Eq. (3) are negative.] Table I shows $\langle R\rangle$ 's for various choices of $\mu_{i}$ 's, $p_{\mu_{i}}$ 's, $Y_{\max }$, and $\ell_{\max }$ using either unbiased or biased bases selection when $e_{\max }=1 \%$. (Note that the intensities and probabilities of the two $k=3$ cases in the table are adapted from actual experiments [20,21].) As expected, the general trend is that the higher the value of $k$, the higher the average key rate in the infinite $\ell_{\max }$ limit. The increase in $\langle R\rangle$ by using six photon intensities can be as high as $77 \%$. Whereas for $\ell_{\text {raw }}=10^{9}$ $\left(10^{10}\right)$, using four (five) types of photon intensities performs better because finite-size fluctuations on $Q_{\mathrm{B}, \mu_{i}}$ 's and $E_{\mathrm{B}, \mu_{i}}$ 's are relatively smaller. The increase in $\langle R\rangle$ in these cases is at least $26 \%(12 \%)$ using unbiased (biased) bases selection. Among the cases with the same $k$, Table I suggests that those using evenly distributed $\mu_{i}$ 's in $[0,1]$ in general have a slightly higher $\langle R\rangle$. It is instructive to know why. Lastly, I find that the relative errors of the bounds $Y_{\mathrm{B}, 1}$ and $Y_{\mathrm{Z}, 1} e_{\mathrm{Z}, 1}$ from their actual values for cases $\mathrm{A}-\mathrm{H}$ reduces from $\approx 10^{-2}$ to $\approx 10^{-4}$ when $k$ increases from 3 to 6 in the infinite raw key length limit. An explanation is given in the Appendix.

In summary, I demonstrated the effectiveness of using more than three photon intensities, with several close to 1 intensities used with significant chance, to obtain a high provably secure key rate through tighter bounds on $Y_{\mathrm{B}, 1}$ and $e_{\mathrm{Z}, 1}$ and at the same time a higher value of $\langle\mu \exp (-\mu)\rangle$. The initial study here shows an average of at least $20 \%$ improvement on the
TABLE I. Average key rates for different decoy states with $e_{\max }=1 \%$ for (a) $p_{\mathrm{x}}=0.50$ and (b) $p_{\mathrm{X}}=0.75$ over a sample of $10^{6}$ different $Y_{\mathrm{B}, m}$ 's and $E_{\mathrm{B}, m}$ 's. For decoy parameters in case $\mathrm{A}, \mu_{i}$ 's $=\left(0.66,0.05,10^{-6}\right)$ and $p_{\mu_{i}}$ 's $=(1 / 3,1 / 3,1 / 3)$. Corresponding parameters for the other cases are B: $\left(0.8,0.1,10^{-6}\right)$ and $(1 / 2,1 / 4,1 / 4) ; \mathrm{C}:\left(0.8,0.5,0.35,10^{-6}\right)$ and $(1 / 2,1 / 6,1 / 6,1 / 6)$; D: $\left(1.0,0.67,0.33,10^{-6}\right)$ and $(1 / 2,1 / 6,1 / 6,1 / 6)$; E: $\left(0.8,0.65,0.5,0.35,10^{-6}\right) \quad$ and $(1 / 2,1 / 8,1 / 8,1 / 8,1 / 8)$; F: $\left(1,0.75,0.5,0.1,10^{-6}\right)$ and $(1 / 2,1 / 8,1 / 8,1 / 8,1 / 8) ; \quad G$ : $\left(1,0.8,0.65,0.5,0.35,10^{-6}\right)$ and $(0.5,0.1,0.1,0.1,0.1,0.1)$; and $\mathrm{H}$ : $\left(1,0.8,0.6,0.4,0.2,10^{-6}\right)$ and $(0.5,0.1,0.1,0.1,0.1,0.1)$.

(a) $p_{\mathrm{X}}=0.50$

\begin{tabular}{ccccccc}
\hline & & \multicolumn{5}{c}{ Average key rate when $\ell_{\text {raw }}=$} \\
\cline { 3 - 7 }$Y_{\max }$ & & $k$ & $10^{9}$ & $10^{10}$ & $10^{11}$ & $\infty$ \\
\hline \multirow{2}{*}{0.1} & A & 3 & $2.4 \times 10^{-4}$ & $2.9 \times 10^{-4}$ & $3.2 \times 10^{-4}$ & $3.4 \times 10^{-4}$ \\
& B & 3 & $4.4 \times 10^{-4}$ & $5.1 \times 10^{-4}$ & $5.4 \times 10^{-4}$ & $5.6 \times 10^{-4}$ \\
& C & 4 & $4.1 \times 10^{-4}$ & $5.7 \times 10^{-4}$ & $6.6 \times 10^{-4}$ & $7.1 \times 10^{-4}$ \\
& D & 4 & $5.6 \times 10^{-4}$ & $6.9 \times 10^{-4}$ & $7.4 \times 10^{-4}$ & $7.7 \times 10^{-4}$ \\
& E & 5 & $2.2 \times 10^{-4}$ & $5.2 \times 10^{-4}$ & $7.2 \times 10^{-4}$ & $8.7 \times 10^{-4}$ \\
& F & 5 & $5.3 \times 10^{-4}$ & $7.3 \times 10^{-4}$ & $8.2 \times 10^{-4}$ & $8.9 \times 10^{-4}$ \\
& G & 6 & $2.2 \times 10^{-5}$ & $1.9 \times 10^{-4}$ & $5.0 \times 10^{-4}$ & $9.9 \times 10^{-4}$ \\
& H & 6 & $2.1 \times 10^{-4}$ & $5.1 \times 10^{-4}$ & $7.4 \times 10^{-4}$ & $9.4 \times 10^{-4}$ \\
0.01 & A & 3 & $2.4 \times 10^{-5}$ & $2.9 \times 10^{-5}$ & $3.2 \times 10^{-5}$ & $3.3 \times 10^{-5}$ \\
& B & 3 & $4.5 \times 10^{-5}$ & $5.1 \times 10^{-5}$ & $5.4 \times 10^{-5}$ & $5.6 \times 10^{-5}$ \\
& C & 4 & $4.1 \times 10^{-5}$ & $5.7 \times 10^{-5}$ & $6.6 \times 10^{-5}$ & $7.1 \times 10^{-5}$ \\
& D & 4 & $5.6 \times 10^{-5}$ & $6.8 \times 10^{-5}$ & $7.4 \times 10^{-5}$ & $7.7 \times 10^{-5}$ \\
& E & 5 & $2.3 \times 10^{-5}$ & $5.2 \times 10^{-5}$ & $7.2 \times 10^{-5}$ & $8.6 \times 10^{-5}$ \\
F & 5 & $5.3 \times 10^{-5}$ & $7.3 \times 10^{-5}$ & $8.2 \times 10^{-5}$ & $8.8 \times 10^{-5}$ \\
& G & 6 & $2.2 \times 10^{-6}$ & $1.9 \times 10^{-5}$ & $5.0 \times 10^{-5}$ & $9.8 \times 10^{-5}$ \\
H & 6 & $2.1 \times 10^{-5}$ & $5.1 \times 10^{-5}$ & $7.4 \times 10^{-5}$ & $9.3 \times 10^{-5}$
\end{tabular}

(b) $p_{\mathrm{x}}=0.75$

Average key rate when $\ell_{\text {raw }}=$

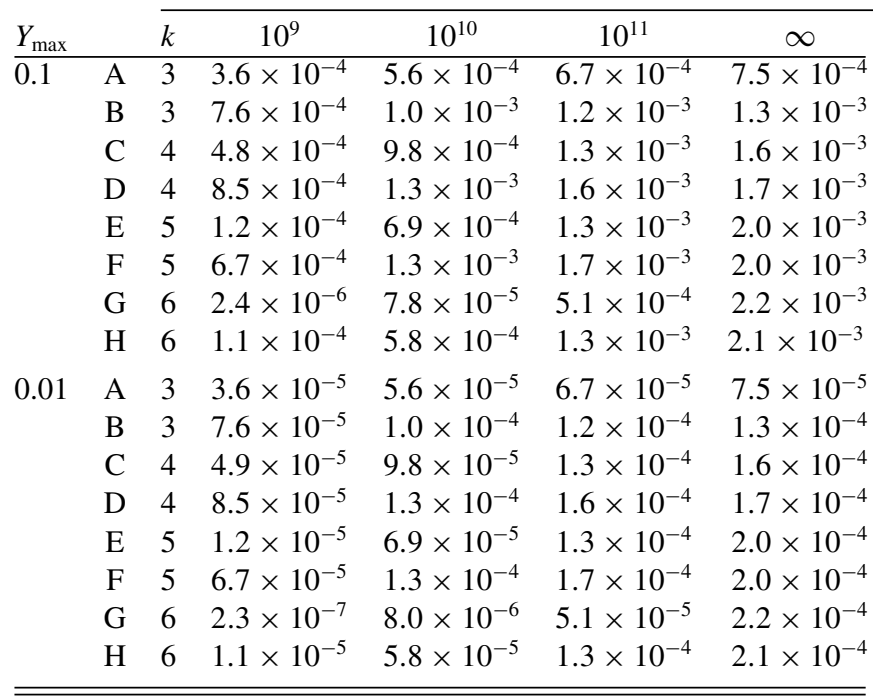

average key rate. It is instructive to further optimize the choice of intensity parameters $\mu_{i}$ and $p_{\mu_{i}}$ to see how far one can go.

This work is supported by the Research Grant Council (RGC) Grant No. 17304716 of the Hong Kong SAR Govern- 
ment. I would like to thank X. D. Cui and Z.-Q. Yin for their discussions on laser intensity fluctuation.

\section{APPENDIX: THE SIGNS OF $C_{0 j}$ AND $C_{1 j}$}

From Eq. (10),

$$
C_{m+1, j}=\frac{(-1)^{k-m} m !}{j !} \sum_{i=1}^{k} \frac{\mu_{i}^{j} S_{i m}}{\prod_{t \neq i}\left(\mu_{i}-\mu_{t}\right)}
$$

for all $0 \leqslant m \leqslant k-1$. From Eq. (11), only the first two terms in the above sum contain the factor $\left(\mu_{1}-\mu_{2}\right)$ in their denominators; and by summing these two terms, this factor is canceled if $j \geqslant 0$. Note further that $C_{m+1, j}$ is a symmetric function of variables $\mu_{i}$. Hence, $C_{m+1, j}$ is a homogeneous polynomial of degree $\leqslant j-m$ in the case of $j \geqslant m$. If $j \geqslant 1$, terms in the degree $j$ homogeneous polynomial $C_{1 j}$ contain the common factor $\prod_{n=1}^{k} \mu_{n}$. Therefore, $C_{1 j}$ is actually a constant whenever $1 \leqslant j<k$. Whereas, for $j \geqslant k$, by counting the leading power term for $\mu_{1}$ in $C_{1 j}$, I conclude that $C_{1 j}$ is a homogeneous polynomial of degree $j$. Then, by fixing $\mu_{2}, \mu_{3}, \ldots, \mu_{k}$ and considering the series expansion of $1 /\left(\mu_{1}-\mu_{n}\right)$ 's in the large $\mu_{1}$ limit, I get

$$
\begin{aligned}
C_{1 j}= & \frac{(-1)^{k}}{j !}\left(\prod_{t=1}^{k} \mu_{t}\right)\left[\mu_{1}^{j-k} \prod_{r=2}^{k}\left(1+\frac{\mu_{r}}{\mu_{1}}+\frac{\mu_{r}^{2}}{\mu_{1}^{2}}+\cdots\right)\right. \\
& \left.+f\left(\mu_{2}, \mu_{3}, \ldots, \mu_{k}\right)\right]
\end{aligned}
$$

for some function $f$ independent of $\mu_{1}$. As $C_{1 j}$ is a homogeneous polynomial, by equating terms in powers of $\mu_{1}$, I arrive at

$$
C_{1 j}=\frac{(-1)^{k}}{j !}\left(\prod_{t=1}^{k} \mu_{t}\right)_{\substack{t_{1}+\cdots+t_{k}=j-k, t_{1}, \ldots, t_{k} \geqslant 0}} \mu_{1}^{t_{1}} \mu_{2}^{t_{2}} \cdots \mu_{k}^{t_{k}}
$$

for all $j \geqslant k$. As all $\mu_{i}$ 's are non-negative, I conclude that $C_{1 j} \geqslant 0$ if $k$ is even and $C_{1 j} \leqslant 0$ if $k$ is odd with equality holds if and only if the least photon intensity $\mu_{n}=0$.

The same argument leads to

$$
\begin{aligned}
C_{2 j}= & \frac{(-1)^{k-1}}{j !}\left(\sum_{t=1}^{k} \mu_{1} \cdots \mu_{t-1} \mu_{t+1} \cdots \mu_{k}\right) \\
& \times \sum_{\substack{t_{1}+\cdots+t_{k}=j-k, t_{1}>0, t_{2}, \ldots, t_{k} \geqslant 0}} \mu_{1}^{t_{1}} \mu_{2}^{t_{2}} \cdots \mu_{k}^{t_{k}}+f^{\prime}\left(\mu_{2}, \mu_{3}, \ldots, \mu_{k}\right)
\end{aligned}
$$

for all $j \geqslant k$. Here the function $f^{\prime}$ can be found by recursively expanding Eq. (A1) in the same way as Eq. (A2) in powers of $\mu_{2}$ but with $\mu_{1}$ set to 0 , and then in powers of $\mu_{3}$ with $\mu_{1}$ and $\mu_{2}$ set to 0 , and so on. Although the resultant expression is very complicated, it is easy to see that $C_{2 j}<0$ if $k$ is even and $C_{2 j}>0$ if $k$ is odd. Interested readers may apply this method to find explicit expressions for $C_{m+1, j}$ 's for $m>2$.

Finally, suppose the bound of $Y_{\mathrm{B}, 1}$ or $Y_{\mathrm{Z}, 1} e_{\mathrm{Z}, 1}$ is obtained from a set $K$ of $k^{\prime}$ different photon intensities. Then, the relative error of the bound of $Y_{\mathrm{B}, 1}$ from its actual value is about $\left|C_{2 k^{\prime}}\right|$. If one of the used intensities $\mu_{r} \approx 0$, then Eq. (A4) gives $\left|C_{2 k^{\prime}}\right| \approx$ $\prod_{t \in K \backslash\{r\}} \mu_{t} / k^{\prime}$ !. For the choice of parameters in Table I, $\left|C_{2 k^{\prime}}\right|$ reduces from about $2 \%$ to $0.03 \%$, which is consistent with the numerical findings. More importantly, this estimation justifies the use of the least $2\lfloor k / 2\rfloor$ or $2\lfloor(k-1) / 2\rfloor+1$ intensities to bound the variables in the main text.
[1] V. Scarani, H. Bechmann-Pasquinucci, N. J. Cerf, M. Dušek, N. Lütkenhaus, and M. Peev, Rev. Mod. Phys. 81, 1301 (2009).

[2] X.-B. Wang, Phys. Rev. Lett. 94, 230503 (2005).

[3] H.-K. Lo, X. Ma, and K. Chen, Phys. Rev. Lett. 94, 230504 (2005).

[4] X. Ma, Chi-Hang Fred Fung, F. Dupuis, K. Chen, K. Tamaki, and H.-K. Lo, Phys. Rev. A 74, 032330 (2006).

[5] D. Bunandar, Z. Zhang, J. H. Shapiro, and D. R. Englund, Phys. Rev. A 91, 022336 (2015).

[6] Charles Ci Wen Lim, M. Curty, N. Walenta, F. Xu, and H. Zbinden, Phys. Rev. A 89, 022307 (2014).

[7] X. Ma, B. Qi, Y. Zhao, and H.-K. Lo, Phys. Rev. A 72, 012326 (2005).

[8] C. H. Bennett and G. Brassard, in Proceedings of the IEEE International Conference on Computers, Systems and Signal Processing (IEEE, New York, 1984), pp. 175-179.

[9] K. Brádler, M. Mirhosseini, R. Fickler, A. Broadbent, and R. Boyd, New J. Phys. 18, 073030 (2016).

[10] H. F. Chau, C. Wong, T. Huang, and Q. Wang, Provably secure key rate analysis of finite-key-length qudit-based decoy state quantum key distribution (unpublished).
[11] R. Renner, Security of QKD, Ph.D. thesis, ETH, 2005 (Diss. ETH No. 16242).

[12] B. Kraus, N. Gisin, and R. Renner, Phys. Rev. Lett. 95, 080501 (2005).

[13] R. Renner, N. Gisin, and B. Kraus, Phys. Rev. A 72, 012332 (2005).

[14] W. Hoeffding, J. Am. Stat. Assoc. 58, 13 (1963).

[15] Chi-Hang Fred Fung, X. Ma, and H. F. Chau, Phys. Rev. A 81, 012318 (2010).

[16] M. Hayashi, New J. Phys. 9, 284 (2007).

[17] T. Schmitt-Manderbach, H. Weier, M. Fürst, R. Ursin, F. Tiefenbacher, T. Scheidl, J. Perdigues, Z. Sodnik, C. Kurtsiefer, J. G. Rarity, A. Zeilinger, and H. Weinfurter, Phys. Rev. Lett. 98, 010504 (2007).

[18] Y. Liu, T.-Y. Chen, J. Wang, W.-Q. Cai, X. Wan, L.-K. Chen, J.-H. Wang, S.-B. Liu, H. Liang, L. Yang, C.-Z. Peng, K. Chen, Z.-B. Chen, and J.-W. Pan, Opt. Express 18, 8587 (2010).

[19] M. Lucamarini, K. A. Patel, J. F. Dynes, B. Fröhlich, A. W. Sharpe, A. R. Dixon, Z. L. Yuan, R. V. Penty, and A. J. Shields, Opt. Express 21, 24550 (2013). 
[20] S.-K. Liao et al., Nature (London) 549, 43 (2017).

[21] S. Wang, Z.-Q. Yin, H. F. Chau, W. Chen, G.-C. Guo, and Z.-F. Han, Quant. Sci. Tech. 3, 025006 (2018).
[22] D. E. Knuth, The Art of Computer Programming: Volume 1, 2nd ed. (Addison-Wesley, Reading, MA, 1973), Ex. 1.2.3.40 on p. 36 .

[23] N. Walenta, T. Lunghi, O. Guinnard, R. Houlmann, H. Zbinden, and N. Gisin, J. Appl. Phys. 112, 063106 (2012). 